\title{
Echocardiographic evaluation of diastolic dysfunction in young and healthy patients with psoriasis: A case-control study
}

\author{
Elio Gorga', Marta Scodro', Francesca Valentini', Renzo D’Ortona', Mariachiara Arisi², \\ Edoardo Sciatti', Ivano Bonadei', Valentina Regazzoni', Enrico Vizzardi1, Marco Metra1, \\ Piergiacomo Calzavara Pinton² \\ 1 Cardiology, Department of Medical and Surgical Specialties, Radiological Sciences, and Public Health, \\ University of Brescia \\ 2 Department of Dermatology, Spedali Civili of Brescia, University of Brescia, Italy
}

\begin{abstract}
Psoriasis is a systemic inflammatory disease with a great prevalence in general population. The inappropriate activation of the cellular immune system has been hypothesized to be an independent cardiovascular risk factor, given the higher incidence of cardiovascular disorders in psoriatic patients. Echocardiographic abnormalities have been demonstrated too: the aim of our study was to evaluate the presence of preclinical cardiac dysfunction in a cohort of psoriatic patients without cardiovascular risk factors. We enrolled 52 patients with the diagnosis of chronic plaque psoriasis, compared with a control group not affected by any relevant systemic diseases and inflammatory disorders. In all patients and control group, echocardiographic conventional and tissue Doppler (TDI) studies were conducted. The analysis of echocardiographic parameters revealed normal dimension, mass and systolic
\end{abstract}

Corresponding author: Edoardo Sciatti, Cardiology, Department of Medical and Surgical Specialties, Radiological Sciences, and Public Health, University of Brescia, Piazza del Mercato 15, 25121 Brescia. Tel. +39.030.2988.1. E-mail: edoardo.sc@tin.it

Key words: Psoriasis; diastolic dysfunction; cardiovascular risk; atherosclerosis; echocardiography.

Contributions: EG, MA, ES, VR, data collection; MS, FV, RDO, manuscript drafting; IB, EV, MM, PCP, reviewing article. All authors read and approved the final version of the manuscript to be published.

Conflict of interest: The authors declare no conflict of interest.

Received for publication: 26 March 2018

Accepted for publication: 18 July 2018

(C) Copyright E. Gorga et al., 2018

Tipografia PI-ME Editrice, Italy

Monaldi Archives for Chest Disease 2018; 88:934

doi: 10.4081/monaldi.2018.934

This article is distributed under the terms of the Creative Commons Attribution Noncommercial License (by-nc 4.0) which permits any noncommercial use, distribution, and reproduction in any medium, provided the original author(s) and source are credited. function of the left ventricle. Left ventricular diastolic dysfunction was found in $36.5 \%$ patients in the psoriasis group versus $0 \%$ in control group, and significant reduction of the E/A ratio was found also for the right ventricle. A significant increase of mitral regurgitation has been found in psoriatic patients $(\mathrm{p}=0.005)$. The early recognition of cardiovascular pre-clinic disease in psoriatic patients may guide a strict follow up and an early treatment, potentially improving cardiovascular prognosis.

\section{Introduction}

Psoriasis is a systemic inflammatory disease that affects $1.5-3 \%$ of the general population [1]. Pathophysiology is multifactorial, depending on an association between genetic predisposition and environmental factors that determine an inappropriate activation of the cellular immune system [2].

The role of psoriasis as an independent cardiovascular risk factor (CVRF) is debated: a meta-analysis by Gaeta et al. based on 13 studies including patients with psoriasis showed a significant $24 \%$ excess risk of overall cardiovascular diseases (CVDs) in patients with psoriasis as compared to controls ( $\mathrm{RR}=1.24(1.18-1.31) ; \mathrm{P}=0.0001$ ) and this risk appears to be independent of smoking, obesity and hyperlipidemia [3]. Several previous reports have also showed a higher incidence of cardiovascular disorders such as arterial hypertension, stroke, coronary artery diseases, heart failure, arrhythmias and valvular disorders in patients with psoriasis than in general population and this association was independent from traditional CVRF [4,5]. Some authors have suggested that the association between psoriasis and CVDs depends on a common chronic inflammatory pathway [6]. Psoriasis is characterized by an overexpression of pro-inflammatory cytokines, such as interleukins, TNF- $\alpha$ and interferon- $\beta$ which play an important role in determining endothelial dysfunction, atherosclerosis promotion and cardiomyocytes toxicity. Psoriasis has been also associated with echocardiographic abnormalities: a study by Biyik et al., including 216 psoriatic patients compared with healthy controls, revealed a significant increase of cardiac abnormalities such as left ventricular hypertrophy, altered left ventricular wall motion and valvular diseases, especially mitral and tricuspidal valve prolapse, and left ventricular diastolic dysfunction in psoriasis group [7]. Diastolic dysfunction has been also observed in patients without cardiovascular risk factors [8].

The aim of our study was to evaluate the presence of preclinical cardiac dysfunction in a cohort of psoriatic patients without CVRFs. 


\section{Materials and Methods}

\section{Study patients}

This was a case-control study to evaluate the presence of echocardiographic anomalies in a population of psoriatic patients without cardiovascular risk factors.

We enrolled patients affected by plaque psoriasis followed-up at the Dermatology Unit of University and Civil Hospital of Brescia. The inclusion criteria were a clinical diagnosis of chronic plaque psoriasis, lasting at least 6 months, in a stable phase of the disease and a psoriasis area severity index (PASI) of at least 10. The type of treatment was not an exclusion criterion. However, it should not have been changed in the 4 weeks preceding the assessment visit. Controls, matched for sex and age, were recruited among healthcare workers of the hospital. Exclusion criteria for both patients and controls were age $<18$ years, pregnancy, lactation, detection of even a single cardiovascular risk factor (as hypertension, diabetes or dyslipidemia) or any known cardiovascular disease (any coronary syndrome, valvular disease with severity greater than medium, cardiomyopathies, heart rhythm anomalies); familiar history of cardiac or cerebral diseases as of any systemic peripheral arterial disease, in first degree relatives; tobacco use, considering both cigarette and cigar; dyslipidemia diagnosed following cut of value of the European Society of Cardiology (LDL-c $>140 \mathrm{mg} / \mathrm{dL}$, tot-c $>200 \mathrm{mg} / \mathrm{dL}$, TGL $>180 \mathrm{mg} / \mathrm{dl}$, HDL-c $<40 \mathrm{mg} / \mathrm{dL}$ ); diabetes mellitus or impaired fasting glycaemia; obesity (BMI $>30$ ) or underweight (BMI< 18); hypertension, diagnosed by values of arterial pressure more than $140 / 80 \mathrm{mmHg}$ at the clinical evaluation or by the use of antihypertensive drugs. Controls were not affected by any relevant systemic diseases and inflammatory skin or systemic disorders.

The study was conducted in the Cardiology Unit of University and Civil Hospital of Brescia, Italy. We certified that the study complied with the ethical standards of the Declaration of Helsinki [9] and all patients gave their written, informed consent to participate.

\section{Study protocol}

Patients eligible for the study underwent a complete clinical assessment, electrocardiogram and transthoracic echocardiography.

They underwent 2-dimensional and Doppler transthoracic echocardiography. Based on our previous study [10], echocardiograms were performed using Vivid 7 (GE) with a 3.5 transducer. Subjects were examined in the left decubitus position according to the standardization of the American Society of Echocardiography. Left ventricular dimensions and ejection fraction (LVEF) were estimated according to LV volumes evaluated by biplane Simpson's method [11].

Mitral and tricuspid valve regurgitation grade were assessed semiquantitatively according to the current guidelines and classified as mild, moderate and severe [12]. By placing the 2-mm PWD at the mitral inflow, the early diastolic (E) and atrial (A) velocities were measured and
E/A ratio was calculated. The deceleration time (DT) was measured according to the recommendations of the American Society of Echocardiography [13]. Myocardial systolic and diastolic velocities were recorded using the PW tissue Doppler Imaging (TDI). Systolic (S'), early (E') and late $\left(\mathrm{A}^{\prime}\right)$ diastolic waves velocities were measured by placing the sample volume, underneath the mitral annulus, on the lateral and on the septal myocardial wall of the $\mathrm{LV}$ from the apical 4-chamber view and on the tricuspidal lateral annulus. The E/E' ratio was also obtained; we used the average of E' left sides velocities for the prediction of LV filling pressures [14]. According to the recommendations of the American Society of Echocardiography [14], LV diastolic dysfunction was defined as septal E' less than $8 \mathrm{~cm} / \mathrm{sec}$ or lateral E' less than $10 \mathrm{~cm} / \mathrm{sec}$; different rates of diastolic dysfunction were classified in mild or grade 1 (impaired relaxion pattern), moderate or grade 2 (pseudonormal filling pattern), and severe or grade 3 (restrictive filling pattern). Conventional PW echocardiography has been used to derive left ventricular and RV myocardial performance indexes (MPIs) as previously described by Tei and colleagues, considering as normal LV MPI less than 0.40 and RV MPI less than 0.30 [15]. Patients had to answer to a specific questionnaire for the assessment of cardiovascular risk factors with the help of trained nurse.

\section{Statistical analysis}

Continuous variables were visually tested for normality using Q-Q plots and expressed as mean \pm standard deviation, while categorical variables as frequency (n) and percentage of the sample. After Levene's test for homoscedasticity, independent samples Welch's $t$-test was performed to analyze the difference between means for continuous variables. The $\chi^{2}$ test was used for assessing differences between proportions. Statistical analysis was performed using IBM SPSS Statistics 20 for Windows (SPSS, Inc., Chicago, IL, USA). All values were two-tailed; statistical significance was set at $\mathrm{p}<0.05$.

\section{Results}

From January 2015 to December 2015 we evaluated 424 patients followed by the Dermatology Unit with a diagnosis of chronic plaque psoriasis. After evaluation for inclusion and exclusion criteria we enrolled 52 patients. The expected 6 months' enrollment period has required an extension due to the difficulty encountered in finding patients corresponding to inclusion and exclusion criteria. Baseline characteristics are shown in Table 1. At study entry, the two groups were balanced with respect to demographic and clinical variables. The analysis of echocardiographic parameters reveals normal dimension, mass and systolic function of the left ventricle, according to the clinical characteristics of patients (Table 2). The only difference in dimensions was telesystolic diameter (DTS), which was larger in cases respect to controls $(\mathrm{p}<0.001)$.

The most interesting result was about left ventricle diastolic dysfunction, defined as $\mathrm{E} / \mathrm{A}<1$ and deceleration time $>220 \mathrm{msec}$ and $\mathrm{E} / \mathrm{E}^{\prime}<10$,

Table 1. Clinical and demographic characteristics of the study population.

\begin{tabular}{lccc} 
Variable & $\begin{array}{c}\text { Group } 1 \\
\text { Psoriasis }(\mathrm{n}=52)\end{array}$ & $\begin{array}{c}\text { Group } 2 \\
\text { Controls }(\mathrm{n}=52)\end{array}$ \\
Age (years) & $44.3 \pm 14.8$ & $39.7 \pm 10.5$ & 0.792 \\
\hline Sex & Male: $27(51.9 \%)$ & Male: $25(48.1 \%)$ & 0.845 \\
\hline SBP $(\mathrm{mmHg})$ & Female: $25(48.1 \%)$ & Female: $27(51.9 \%)$ & \\
\hline DBP $(\mathrm{mmHg})$ & $120.6 \pm 13.2$ & $118.2 \pm 13.0$ & 0.382 \\
\hline Heart rate $(\mathrm{bpm})$ & $73.3 \pm 7.4$ & $70.1 \pm 9.7$ & 0.046 \\
\hline
\end{tabular}


found in 19 (36.5\%) patients in the psoriasis group versus $0 \%$ in control group (Table 3 ). However, left atrial dimensions were not statistically different among the two groups. Differences were also found in right ventricle diastolic function between case and controls (MPI: $0.23 \pm 0.07$ vs $0.41 \pm 0.04, \mathrm{p}<0.01$; E/A $1.27 \pm 0.32$ vs $2.26 \pm 0.90, \mathrm{p}<0.001$ ), but not in systolic function (TAPSE 25,00 mm vs 24,40 mm, p 0.460) (Table 4).
Pulmonary arterial systolic pressure was slightly higher in psoriatic patients then in controls, even without pulmonary hypertension ( $p=0.008$ ). Moreover, considering cardiac valves function, we evidenced a significant increase of mitral regurgitation in the psoriatic cohort: $27 \%$ of psoriatic patient have a mild mitral regurgitation versus $3.8 \%$ of control ( $\mathrm{p}=0.005)$ (Table 5).

Table 2. Echocardiographic characteristics of left ventricle, left atrium, right ventricle.

\begin{tabular}{lccc} 
Variable & $\begin{array}{c}\text { Group } 1 \\
\text { Psoriasis }(\mathbf{n}=52)\end{array}$ & $\begin{array}{c}\text { Group 2 } \\
\text { Controls }(\mathrm{n}=52)\end{array}$ \\
EDD $(\mathrm{mm})$ & $45.6 \pm 5.3$ & $47.1 \pm 3.8$ & 0.102 \\
\hline ESD $(\mathrm{mm})$ & $28.8 \pm 6.0$ & $25.1 \pm 3.5$ & $<0.001$ \\
\hline IVST $(\mathrm{mm})$ & $10.3 \pm 1.1$ & $9.4 \pm 1.1$ & 0.527 \\
\hline PWT $(\mathrm{mm})$ & $8.4 \pm 1.5$ & $8.7 \pm 1.2$ & 0.360 \\
\hline Mass $(\mathrm{g})$ & $166.4 \pm 22.1$ & $127.9 \pm 26.7$ & 0.216 \\
\hline RWT & $0.373 \pm 0.076$ & $0.369 \pm 0.056$ & 0.759 \\
\hline LVEF $(\%)$ & $61.8 \pm 6.5$ & $63.2 \pm 4.5$ & 0.189 \\
\hline LA AP diameter $(\mathrm{mm})$ & $31.5 \pm 4.8$ & $30.4 \pm 2.1$ & 0.164 \\
\hline TAPSE $(\mathrm{mm})$ & $25.0 \pm 4.5$ & $24.4 \pm 3.5$ & 0.460 \\
\hline sPAP $(\mathrm{mmHg})$ & $23.8 \pm 4.2$ & $22.1 \pm 1.7$ & 0.008 \\
\hline RV MPI & $0.235 \pm 0.079$ & $0.420 \pm 0.041$ & $<0.001$ \\
\hline
\end{tabular}

Table 3. Left ventricle diastolic function.

\begin{tabular}{lccc} 
Variable & $\begin{array}{c}\text { Group } 1 \\
\text { Psoriasis }(\mathrm{n}=52)\end{array}$ & $\begin{array}{c}\text { Group 2 } \\
\text { Controls }(\mathrm{n}=52)\end{array}$ \\
$\mathrm{E}(\mathrm{m} / \mathrm{s})$ & $0.70 \pm 0.21$ & $0.68 \pm 0.15$ & 0.708 \\
\hline $\mathrm{A}(\mathrm{m} / \mathrm{s})$ & $0.59 \pm 0.21$ & $0.38 \pm 0.07$ & $<0.001$ \\
\hline E/A & $1.36 \pm 0.62$ & $1.87 \pm 0.61$ & $<0.001$ \\
\hline DT $(\mathrm{msec})$ & $209.9 \pm 63.8$ & $187.5 \pm 56.2$ & 0.060 \\
\hline E' $^{\prime}(\mathrm{m} / \mathrm{s})$ & $0.08 \pm 0.02$ & $0.11 \pm 0.02$ & 0.032 \\
\hline A' $\left.^{\prime} \mathrm{m} / \mathrm{s}\right)$ & $0.05 \pm 0.04$ & $0.08 \pm 0.04$ & 0.040 \\
\hline S' $(\mathrm{m} / \mathrm{s})$ & $0.08 \pm 0.03$ & $0.06 \pm 0.17$ & 0.003 \\
\hline E/E' & $7.00 \pm 6.06$ & $6.34 \pm 1.72$ & 0.452 \\
\hline E'/A' & $1.65 \pm 1.00$ & $1.98 \pm 1.12$ & 0.118 \\
\hline Grade I diastolic dysfunction & $19(36.5 \%)$ & $0(0.0 \%)$ & $<0.001$ \\
\hline - E/A $<1$ & & & \\
\hline - DT $>220$ msec & & \\
\hline - E/e' $<10$ & & \\
\hline
\end{tabular}

Table 4. Right ventricle diastolic function.

\begin{tabular}{lccc} 
Variable & $\begin{array}{c}\text { Group 1 } \\
\text { Psoriasis }(\mathbf{n = 5 2})\end{array}$ & $\begin{array}{c}\text { Group 2 } \\
\text { Controls }(\mathbf{n}=52)\end{array}$ & P \\
$\mathrm{E}(\mathrm{m} / \mathrm{s})$ & $0.40 \pm 0.15$ & $0.64 \pm 0.14$ & $<0.001$ \\
\hline $\mathrm{A}(\mathrm{m} / \mathrm{s})$ & $0.34 \pm 0.14$ & $0.31 \pm 0.10$ & 0.299 \\
\hline $\mathrm{E} / \mathrm{A}$ & $1.27 \pm 0.32$ & $2.26 \pm 0.90$ & $<0.001$ \\
\hline
\end{tabular}

Table 5. Valvular abnormalities.

\begin{tabular}{lccc} 
Variable & $\begin{array}{c}\text { Group 1 } \\
\text { Psoriasis }(\mathbf{n = 5 2})\end{array}$ & $\begin{array}{c}\text { Group 2 } \\
\text { Controls (n=52) }\end{array}$ & P \\
MR & $14(26.9 \%)$ & $2(3.8 \%)$ & 0.005 \\
\hline MVP & $4(7.7 \%)$ & $0(0.0 \%)$ & 0.112 \\
\hline MS & $0(0.0 \%)$ & $0(0.0 \%)$ & - \\
\hline AoR & $5(9.6 \%)$ & $0(0.0 \%)$ & 0.057 \\
\hline AoS & $0(0.0 \%)$ & $0(0.0 \%)$ & - \\
\hline ToEct & $4(7.7 \%)$ & $0(0.0 \%)$ & 0.118 \\
\hline
\end{tabular}

MR, mitral regurgitation; MVP, mitral valve prolapse; MS, mitral stenosis; AoR, aortic regurgitation; AoS, aortic stenosis; AoEct,aortic ectasia; TR, tricuspid regurgitation. 


\section{Discussion}

Chronic inflammation is an established cardiovascular risk factor. The latest ESC guidelines in this regard have included a specific chapter for autoimmune diseases: systemic lupus erythematous, rheumatoid arthritis and psoriasis are in fact identified as diseases associated with an increased risk of myocardial infarction [16]. Psoriasis is an immune-modulated inflammatory disorder [5,17] and precisely this pro-inflammatory state has been linked to the increased incidence of diseases such as atherosclerosis, myocardial infarction, heart failure and cerebrovascular events $[18,19]$. The risk seems to increase with the degree of severity of the disease. Both psoriasis and cardiovascular disease are characterized by a T helper-1 cell mediated immune impairment [20-22] with involvement of cytokines such as interferon- $\gamma$, tumor necrosis factor (TNF)- $\alpha$ [23,24], and interleukin-2 [25]. They stimulate keratinocytes to produce IL-6, IL-7, IL-8, IL-12, IL-15, and IL18 [25], and interferon- $\gamma$ [26] that can stimulate the expression of major histocompatibility complex class II molecules and intracellular adhesion molecule (ICAM)-1 [26,27]. In addition, vascular endothelial growth factor (VEGF), a potent pro-angiogenic, has been found up-regulated both in psoriasis and atherosclerosis $[28,29]$. How this common inflammatory pattern interacts remains poorly understood but we could state that it provides a common background to both diseases.

However, data from many recent trials about the correlation between psoriasis and cardiovascular diseases are inconsistent even because cardiovascular risk factors are highly under-diagnosed and consequently under treated [30].

The aim of our decision to conduct a study on a population without CVRFs was to reduce the possibility of potential confounders. Then, even excluding CVRFs, we found the presence of pre-clinical cardiovascular disease in the majority of asymptomatic psoriatic patients.

Echocardiographic evaluation revealed an increased prevalence of diastolic dysfunction in psoriatic patients compared to control population. Diastolic dysfunction, prevalently represented by abnormalities of early to late ventricular filling velocities ratio (E/A), early flow deceleration time (DT) and isovolumic relaxation time (IVRT), may be the first abnormality in heart disease, which involve not only the left, but also the right ventricle (MPI, E/A).

Alterations of matrix metalloproteinases, related to the enhanced inflammatory pathways, lead to an increased stiffness of the extracellular matrix [31,32]. Oxidative stress could instead act on the structure of cardiomyocytes [33] with consequent alteration of Titin isoform and increased myocardial stiffness [34]. Both these mechanisms, together with subclinical atherosclerosis promoted by the above mentioned dysregulated inflammatory state, are able to explain the incremented stiffness of psoriatic hearts revealed by alterations of diastolic function at echocardiography.

The finding of diastolic dysfunction in young patients (completely free from any known common CV risk factor) sustains the importance of chronic inflammation as an independent CV factor.

This also supports the modern hypothesis of heart failure with preserved ejection fraction as a systemic disease correlated to the presence of metabolic syndrome, hypertension, obesity, atrial fibrillation and renal failure: the common pathway between those diseases appear to be the enhanced inflammatory state.

Our study confirms also the greater presence of valvular defects in psoriatic patients. Mitral valve is the most affected (27\% vs $3.8 \%$; $\mathrm{p}=0.005)$, followed by aortic valve $(9.6 \%$ vs $0 \%, \mathrm{p}=0.05)$. These data agree with what previously emerged in literature: valvular defects were found in a range between $40 \%$ and $26 \%$ compared to 20 to $18 \%$ in control groups $[7,35]$.
Pathophysiology of this alteration remains unknown but an autopsy study of Kakturskii et al. revealed mucoid edema and diffuse mononuclear cell infiltration of the mitral and aortic valves suggesting chronic valvulitis [36].

\section{Conclusions}

Our findings support the importance of chronic inflammation as an independent $\mathrm{CV}$ factor, irrespectively from traditional cardiovascular risk factors, such as hypertension, age and lipid disorders, which, on the other hand, surely act like enhancer of the inflammatory balance.

The early recognition of cardiovascular pre-clinic disease in psoriatic patients, thanks to validated echocardiographic parameters which can be obtained easily in most centers, may guide a strict follow up and an early treatment, potentially improving cardiovascular prognosis.

Moreover, psoriatic patients should be controlled more than normal population: this will be reflected in a more careful management of conditions like hypertension, diabetes and dyslipidemia. The dermatologic surgery should become the preferred place for implementation of this primary prevention strategy, allowing the management of psoriatic patients in a holistic way with a 360 degrees promotion of healthy lifestyle by cooperation between cardiologists and dermatologists.

\section{Limitations}

Our study has several limitations. First the small simple size of recruited patients. Anyway, this is certainly balanced by the accurate selection of patients without cardiovascular risk factors. Our trial has been completed in a single institute and this could have determined selection bias due to unknown specific characteristics of the patients from the Dermatology Unit of University and Civil Hospital of Brescia.

Second, we did not evaluate the prognostic value of diastolic dysfunction. This should be evaluated through a specific prospective study.

\section{References}

1. Griffiths CEMC, Barker JJNWN. Pathogenesis and clinical features of psoriasis. Lancet 2007;370:263-71.

2. Kremers HM, McEvoy MT, Dann FJ, Gabriel SE. Heart disease in psoriasis. J Am Acad Dermatol 2007;57:347-54.

3. Gaeta M, Castelvecchio S, Ricci C, et al. Role of psoriasis as independent predictor of cardiovascular disease: A metaregression analysis. Int J Cardiol. 2013;168:2282-8.

4. Markuszeski L, Bissinger A, Janusz I, et al. Heart rate and arrhythmia in patients with psoriasis vulgaris. Arch Med Res 2007;38:64-9.

5. Gelfand JM, Mehta NN, Langan SM. Psoriasis and cardiovascular risk: strength in numbers, part II. J Invest Dermatol 2011;131:1007-10.

6. Wakkee M, Thio HB, Prens EP, et al. Unfavorable cardiovascular risk profiles in untreated and treated psoriasis patients. Atherosclerosis 2007;190:1-9.

7. Iyik IB, Arin AN, Ozok MAB. Echocardiographic and clinical abnormalities in patients with psoriasis. J Int Med Res 2006;34:632-9.

8. Milaniuk S, Pietrzak A, Mosiewicz B, Mosiewicz J. Influence of psoriasis on circulatory system function assessed in echocardiography. Arch Dermatol Res 2015;307:855-61.

9. [No authors listed]. EEC note for guidance: good clinical practice for trials on medicinal products in the European Community. CPMP Working Party on Efficacy of Medicinal Products. Pharmacol Toxicol 1990;67:361-72. 
10. Vizzardi E, Cavazzana I, Bazzani C et al. Echocardiographic evaluation of asymptomatic patients affected by rheumatoid arthritis. J Investig Med 2012;60:1204-8.

11. Lang RM, Badano LP, Mor-Avi V, et al. Recommendations for cardiac chamber quantification by echocardiography in adults: an update from the American Society of Echocardiography and the European Association of Cardiovascular Imaging. J Am Soc Echocardiogr 2015:28:1-39.

12. Zoghbi WA, Enriquez-Sarano M, Foster E, et al. Recommendations for evaluation of the severity of native valvular regurgitation with two-dimensional and Doppler echocardiography. J Am Soc Echocardiogr 2003;16:777Y802.

13. Quinones MA, Otto CM, Stoddard M, et al. Recommendations for quantification of Doppler echocardiography. A report from the Doppler quantification task force of the nomenclature and standards committee of the American Society of Echocardiography. J Am Soc Echocardiogr 2002;15:167Y184.

14. Nagueh SF, Appleton CP, Gillebert TC, et al. Recommendations for the evaluation of left ventricular diastolic function by echocardiography. J Am Soc Echocardiogr 2009;22:107-33.

15. Tei C, Dujardin KS, Hodge DO, et al. New index of combined systolic and diastolic myocardial performance: a simple and reproducible measure of cardiac function; a study in normals and dilated cardiomyopathy. J Cardiol 1995;26:357-66.

16. Piepoli MF, Hoes AW, Agewall S, et al. European Guidelines on cardiovascular disease prevention in clinical practice The Sixth Joint Task Force of the European Society of Cardiology and Other Societies on Cardiovascular Disease Prevention in Clinical Practice. Eur Heart J 2016;37:2315-81.

17. Mehta NN, Azfar RS, Shin DB, et al. Patients with severe psoriasis are at increased risk of cardiovascular mortality: cohort study using the General Practice Research Database. Eur Heart J 2010;31:1000-6.

18. Gelfand JM, Neimann AL, Shin DB, et al. Risk of myocardial infarction in patients with psoriasis. JAMA 2006;296:1735-41.

19. Hansson GK. Immune mechanisms in atherosclerosis. Arterioscler Thromb Vasc Biol 2001;21:1876-90.

20. Gudjonsson JE, Johnston A, Sigmundsdottir H, Valdimarsson H. Immunopathogenic mechanisms in psoriasis. Clin Exp Immunol 2004;135:1-8.

21. Biedermann T, Rocken M, Carballido JM. TH1 and TH2 lymphocyte development and regulation of TH cell-mediated immune responses of the skin. J Investig Dermatol Symp Proc 2004;9:5-14.
22. Nickoloff BJ, Xin H, Nestle FO, Qin JZ. The cytokine and chemokine network in psoriasis. Clin Dermatol 2007;25:568-73.

23. Hansson GK, Libby P. The immune response in atherosclerosis: a double-edged sword. Nat Rev Immunol 2006;6:508-19.

24. Nickoloff BJ, Xin H, Nestle FO, Qin J-Z. The cytokine and chemokine network in psoriasis. Clin Dermatol 2007;25:568-73.

25. Ghazizadeh R, Shimizu H, Tosa M, Ghazizadeh M. Pathogenic mechanisms shared between psoriasis and cardiovascular disease. Int J Med Sci 2010;7:284-9.

26. Ranjbaran H, Sokol SI, Gallo A, et al. An inflammatory pathway of IFN-gamma production in coronary atherosclerosis. J Immunol 2007;178:592-604.

27. Krueger JG. The immunologic basis for the treatment of psoriasis with new biologic agents. J Am Acad Dermatol 2002;46:1-6.

28. Herrmann J, Lerman L0, Mukhopadhyay D, et al. Angiogenesis in atherogenesis. Arterioscler Thromb Vasc Biol 2006;26:1948-57.

29. Canavese M, Altruda F, Ruzicka T, Schauber J. Vascular endothelial growth factor (VEGF) in the pathogenesis of psoriasis - a possible target for novel therapies? J Dermatol Sci 2010;58:171-6.

30. Kimball AB, Szapary P, Mrowietz U, et al. Underdiagnosis and undertreatment of cardiovascular risk factors in patients with moderate to severe psoriasis. J Am Acad Dermatol 2012;67:76-85.

31. Heerebeek L van, Hamdani N, Handoko ML, et al. Diastolic stiffness of the failing diabetic heart: importance of fibrosis, advanced glycation end products, and myocyte resting tension. Circulation 2008;117:43-51.

32. Gonzalez A, Lopez B, Querejeta R, et al. Filling pressures and collagen metabolism in hypertensive patients with heart failure and normal ejection fraction. Hypertension 2010;55:1418-24.

33. Borbely A, Velden J van der, Papp Z, et al. Cardiomyocyte stiffness in diastolic heart failure. Circulation 2005;111:774-81.

34. Grutzner A, Garcia-Manyes S, Kotter S, et al. Modulation of titinbased stiffness by disulfide bonding in the cardiac titin N2-B unique sequence. Biophys J 2009;97:825-34.

35. Gonzalez-Juanatey C, Amigo-Diaz E, Miranda-Filloy JA, et al. Lack of echocardiographic and Doppler abnormalities in psoriatic arthritis patients without clinically evident cardiovascular disease or classic atherosclerosis risk factors. Semin Arthritis Rheum 2006;35:333-9.

36. Kakturskii LB, Belova EB, Polosova TA, Skatnikova VI. Cardiac lesion in psoriasis. Arkh Patol 2004;66:22-4. 